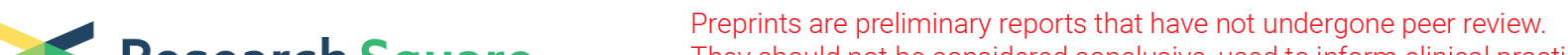 semance \\ IL-21 Rescues The Defect of IL-10-Producing Regulatory B Cells and Improves Allergic Asthma in DOCK8 Deficient Mice
}

\section{Jinqiu Jiang}

Chongqing Key Laboratory of Child Infection and Immunity

\section{Tao Qin}

Chongqing Key Laboratory of Child Infection and Immunity

\section{Liang Zhang}

Chongqing Key Laboratory of Child Infection and Immunity

Qiao Liu

Chongqing Key Laboratory of Child Infection and Immunity

\section{Jiabin Wu}

Chongqing Key Laboratory of Child Infection and Immunity

\section{Rongxin Dai}

Chongqing Key Laboratory of Child Infection and Immunity

\section{Lina Zhou}

Chongqing Key Laboratory of Child Infection and Immunity

\section{Qin Zhao}

Chongqing Key Laboratory of Child Infection and Immunity

\section{Xiaoyan Luo}

Children's Hospital of Chongqing Medical University

\section{Hua Wang}

Children's Hospital of Chongqing Medical University

Xiaodong Zhao ( $\nabla$ zhaoxd530@aliyun.com )

Children's Hospital of Chongqing Medical University https://orcid.org/0000-0003-2054-8711

\section{Research Article}

Keywords: regulatory B cells, IL-10, DOCK8 deficiency, asthma, IL-21

Posted Date: February 15th, 2021

DOI: https://doi.org/10.21203/rs.3.rs-169340/v1 
License: (c) (i) This work is licensed under a Creative Commons Attribution 4.0 International License. Read Full License

Version of Record: A version of this preprint was published at Frontiers in Immunology on November 15th, 2021. See the published version at https://doi.org/10.3389/fimmu.2021.695596. 


\section{Abstract}

Purpose Mutations in human DOCK8 cause a combined immunodeficiency syndrome characterized by allergic diseases such as asthma and food allergy. However, the underlying mechanism is unclear. Regulatory B (Breg) cells that produce IL-10 exert potent immunosuppressive functions in patients with allergic and autoimmune disorders. DOCK8-deficient B cells have been revealed diminished responses to LPS stimulation, suggesting a possible defect in IL-10-producing Breg cells in those with DOCK8 deficiency, which may contribute to allergies.

Methods we collected peripheral blood mononuclear cells from DOCK8-deficient patients and generated a DOCK8KO mouse model to study the effect of DOCK8 deficiency on the Regulatory B cells.

Results We show that DOCK8-deficient patients and DOCK8KO mice harbor quantitative and qualitative defects in IL-10-producing Breg cells; these defects are caused by abnormal DOCK8 ${ }^{-1}$ IL-21-producing $\mathrm{CD} 4^{+} \mathrm{T}$ cells. We found that recombinant murine $(\mathrm{rm}) \mathrm{IL}-21$ restores the function of Bregs both in vitro and in DOCK8KO mice, leading to reduced inflammatory cell infiltration of the lungs in a murine asthma model.

Conclusion Overall, the results provide new insight into the potential design of Breg-based or IL-21-based therapeutic strategies for allergic diseases, including asthma with DOCK8 deficiency.

\section{Introduction}

Mutations in dedicator of cytokinesis 8 (DOCK8) are the major cause of autosomal recessive hyper-IgE syndromes (HIES), which are characterized by combined immunodeficiency and elevated serum IgE levels $[1,2]$. Patients with HIES caused by DOCK8 deficiency are more susceptible to developing allergic diseases (e.g., asthma, food allergies, and atopic dermatitis) than those with HIES caused by STAT3 mutations [3]. However, the mechanism underlying the high incidence of allergy in DOCK8-deficient individuals is not well understood.

DOCK8 functions as a guanine nucleotide exchange factor that is important for actin cytoskeleton rearrangement and optimal STAT3 phosphorylation; it also serves as an adaptor molecule for TLR9MYD88 signaling in B cells [4,5]. Loss-of-function mutations in DOCK8 contribute to impairment of B cell function and of long-lived memory responses [6]. DOCK8-deficient patients show severe reductions in the numbers of circulating $\mathrm{CD} 19^{+} \mathrm{CD} 27^{+}$memory $\mathrm{B}$ cells and $\operatorname{lgD}{ }^{+} \mathrm{CD} 27^{+}$marginal-zone-like (MZ-like) $\mathrm{B}$ cells, along with dysfunctional formation of germinal centers [5, 7].

Regulatory B (Breg) cells in humans and mice are defined as B cells with immunosuppressive capacity associated with secretion of anti-inflammatory cytokines such as TGF- $\beta$, IL-35, and, particularly, IL-10 [810]. Several Breg subsets have been reported with immunoregulatory function through the secretion of IL10 sharing partially overlapping phenotypes, such as mouse $C D 1 \mathrm{~d}^{\text {hi }} \mathrm{CD} 5^{+} \mathrm{B}$ cells (termed B10 cells)[11], marginal zone (MZ) B cells [12], transitional 2-marginal zone precursors (T2-MZP) [10], and human 


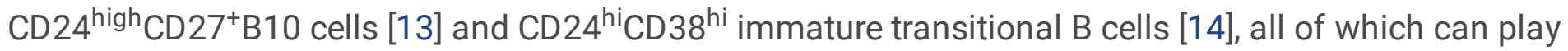
roles in ameliorating inflammatory, autoimmune, and allergic disease such as experimental autoimmune encephalomyelitis, colitis, asthma, and contact hypersensitivity. IL-10-producing Breg cells have direct and indirect suppressive effects on proliferation and cytokine production by effector T cells. However, the precise signaling mechanisms underlying development of IL-10-producing Breg cells is unknown. Previous studies report that BCR-derived signals initiate acquisition of regulatory B10-like competence, and that LPS-induced TLR4 and TLR9 signaling facilitates a transcriptionally active conformation of the IL 10 gene in pro-B10 cells $[15,16]$. In addition, IL-21- and CD40-dependent cognate interactions with $T$ cells are required to generate fully functional mouse B10 cells [17]. DOCK8-deficient B cells show diminished responses to LPS stimulation, suggesting a possible defect in IL-10-producing Breg cells in those with DOCK8 deficiency, which may contribute to allergies.

In this study, we generated DOCK8 KO mice using the TALEN technique; these mice harbor a frameshift mutation in the first exon of dock8, which mirrors human disease because most of the DOCK8-deficient patients are caused by the frame shift or gene deletion in different exons of the DOCK8 gene instead of by point mutation. We found that DOCK8-deficient patients and DOCK8KO mice have quantitative and qualitative defects in IL-10-producing Breg cells due to abnormalities in DOCK8 ${ }^{-/-} \mathrm{IL}-21$-producing CD4 ${ }^{+} \mathrm{T}$ cell populations. We also found that IL-21 rescued the function of Bregs in DOCK8 KO mice and alleviated inflammatory infiltration in a murine asthma model.

\section{Materials And Methods}

\section{Patients}

Three Chinese patients with mutations in the DOCK8 gene were enrolled in the study. All patients were admitted to the Children's Hospital of Chongqing Medical University. Diagnosis of the patients was as previously described [18]. Three age-matched subjects were enrolled as healthy controls (HCs). Informed consent to participate in the study was provided by the patients' families, and the study was approved by the Medical Ethics Committee of Children's Hospital of Chongqing Medical University.

\section{Mice}

DOCK8 KO mice were generated using the TALEN technique (Shanghai Biomodel Organism Science \& Technology Development Co., Ltd). The first exon of DOCK8 was chosen for TALEN-induced mutagenesis; absence of a $45 \mathrm{bp}$ sequence from the coding frame of exon 1 introduced a reading frame shift into the DOCK8 gene [19]. DOCK8 was genotyped by PCR using the following primer pair: sense, 5'GGGGGATCCCCTGCGGCCGGCGACTCTGA-3', and antisense, 5'GGGGAATTCGAAGCGGGGAAGGCAATGATGACA-3'. PCR products amplified from F0 generation mouse tail tissue were purified, cloned, and sequenced to identify positive founder mice with the DOCK8 protein harboring the frame shift. Positive F0 generation mice were crossed with C57BL/6J mice and the genotype of the offspring was confirmed by PCR, cloning, and sequencing. CD $4 K O$ and CD $45.1^{+}$C57BL/ 6 
mice were obtained from the Jackson Laboratory. C57BL/ 6 mice were purchased from the Laboratory Animal Center, Chongqing Medical University. All mice were aged 6 to 10 weeks at the time of the experiments and were housed in specific pathogen-free animal facilities. Data were obtained from three or more mice per group. All animal experiments were reviewed and approved by the Institutional Animal Care and Usage Committee of Children's Hospital of Chongqing Medical University.

\section{Flow cytometry and phosphorylation analyses}

Heparinized blood was obtained from patients and from age-matched HC subjects. Peripheral blood mononuclear cells (PBMCs) were isolated by Ficoll density gradient centrifugation and cell numbers were counted in a hemocytometer. Flow cytometry was performed using a FACSCanto II cytometer (BD Biosciences, San Jose, Calif). Briefly, PBMCs were stained with anti-human CD19-APC, anti-human CD24PE, anti-human CD27-BV450, anti-human CD38-PerCP-cy5.5, or anti-human IgD-BV510. Mononuclear cells isolated from the spleen of DOCK8 KO and WT mice were stained with the following antibodies: antiCD19 FITC, anti-CD5 PE, and anti-CD1d APC (CD1 d ${ }^{\text {hi }}$ CD $5^{+}$B cells); anti-CD19 FITC, anti-CD23 PE, antiCD21 APC, and anti-CD24 Percp-cy5.5 (T2-MZP cells) (all antibodies were from BioLegend, CA). Mononuclear cells isolated from spleen cells of chimera mice were stained with anti-CD45.1 BV510, CD45.2 FITC, anti-CD19 APC, and anti-IL-10 PE (BioLegend, CA). Mononuclear cells isolated from the spleen of CD4 KO mice were stained with anti-CD19 FITC or APC, anti-CD5 PE, anti-CD1d APC, anti-CD23 PE, anti-CD21 APC, anti-CD24 Percp-cy5.5, or anti-IL-10 PE (BioLegend, CA). To detect phosphorylation, single-cell suspensions of splenocytes were stimulated for $3 \mathrm{~h}$ with lipopolysaccharide (LPS; $10 \mu \mathrm{g} / \mathrm{mL}$, Sigma, St. Louis) or for 30 min with rmlL-21 (100 ng/mL; R\&D Systems). Cells were then fixed and permeabilized with BD Phosflow Lyse/Fix Buffer and Perm Buffer III (BD Biosciences), respectively. Finally, cells were stained with anti-B220 FITC, anti-CD5 BV421, anti-CD1d APC, or anti-pY727 PE. All data were analyzed using FlowJo software.

\section{B cell stimulation}

PBMCs isolated from patients or HCs were resuspended (at $2 \times 10^{6} \mathrm{cells} / \mathrm{mL}$ ) in 48-well flat-bottom plates in culture medium and stimulated for $7 \mathrm{~h}$ with LPS (10 $\mathrm{gg} / \mathrm{mL}$; Sigma, St. Louis), phorbol 12-myristate 13acetate (PMA, $20 \mathrm{ng} / \mathrm{mL}$; Sigma, St. Louis), ionomycin $(1 \mu \mathrm{g} / \mathrm{mL}$; Sigma, St. Louis), and brefeldin A (BFA, $1 \times$ solution $/ \mathrm{mL}$; BioLegend, $\mathrm{CA}$ ) before staining and flow cytometry analysis. Next, the cells were harvested and washed twice with PBS. Single-cell suspensions were then stained for 20 min on ice with predetermined optimal concentrations of anti-CD19 APC (BioLegend, CA). Stained cells were washed twice with PBS before fixation and permeabilization in Fixation and Permeabilization Buffer (BioLegend, CA). Finally, cells were stained for 30 min with anti-IL-10 PE (BioLegend, CA). To detect B10 cells in mice, single-cell suspensions of splenocytes were stimulated for $5 \mathrm{~h}$ with LPS, PMA ( $50 \mathrm{ng} / \mathrm{mL})$, ionomycin $(500 \mathrm{ng} / \mathrm{mL})$, and BFA, followed by staining with anti-CD19 APC (BioLegend, CA) and anti-IL-10 PE (BioLegend, CA). For co-culture experiments, splenic B cells were purified using an EasySep ${ }^{\text {TM }}$ Mouse $B$ Cell Isolation Kit (STEMCELL Technologies, Canada). The cells $\left(2 \times 10^{6}\right.$ cells $\left./ \mathrm{mL}\right)$ were then incubated for $48 \mathrm{~h}$ with mouse rmIL-21 (100 ng/mL, R\&D Systems). After culture for $48 \mathrm{~h}$, the IL-10 concentration in the 
supernatant was measured by ELISA (BioLegend, CA), and B10 cells was measured by flow cytometry analysis as described above.

\section{Generation of bone marrow chimeras}

Bone marrow was collected from DOCK8 ${ }^{-/-}\left(\mathrm{CD} 45.2^{+}\right)$mice and from C57BL/ 6 wild-type (CD $\left.45.1^{+}\right)$mice. For each chimera, CD45.2 ${ }^{+}$wild-type or DOCK8 KO bone marrow cells plus CD $45.1^{+}$bone marrow cells ( 4 $\times 10^{6}$ cells in a 1:1 mixture) were transferred intravenously into lethally-irradiated (two doses of 550 rads each) wild-type CD $45.1^{+}$recipients. Recipient mice were allowed 8 weeks to reconstitute prior to challenge with ovalbumin (OVA).

\section{Generation of OVA-induced allergic asthma model mice and nasal administration of recombinant IL-21}

OVA-induced allergic asthma was elicited by sensitization with chicken OVA ( $5 \mu$ g, intraperitoneally (i.p.); Sigma-Aldrich) emulsified in $200 \mathrm{ml}$ Imject Alum (Thermo Fisher Scientific) on Day 0, followed by two oropharyngeal aspiration challenges (on Days 14 and 21) with 1.5\% OVA dissolved in $50 \mathrm{ml} \mathrm{PBS}$. Control mice received only PBS. Mice were harvested $72 \mathrm{~h}$ after the second challenge[20]. Some mice received 20 ng of rmlL-21 (R\&D Systems) into the nostrils (daily on Days 15-17, 18-20, or 15-20). This 3 day protocol was decided by conducting preliminary time-course experiments. The dose of rmIL-21 was obtained from a previous study[21]. As a negative control, groups of mice received neither sensitization nor challenge treatment, except for the final challenge on Day 21.

\section{Adoptive cell transfer}

Naïve splenic CD $4^{+} T$ cells isolated from DOCK8 KO or wild-type mice were purified with the EasySep ${ }^{\mathrm{TM}}$ Mouse Naïve CD $4^{+} \mathrm{T}$ Cell Isolation Kit (STEMCELL Technologies, Canada). Next, $5 \times 10^{6}$ naïve CD $4^{+} \mathrm{T}$ cells were adoptively transferred into CD4KO mice via intravenous injection 1 day before immunization with OVA. OVA immunization was performed as described above. Recipient mice were analyzed on Day 21 post-OVA immunization.

\section{Histopathological analysis}

Lung tissue was harvested and fixed for $24 \mathrm{~h}$ in $10 \%$ formalin and then embedded in paraffin. Sections (4 $\mu \mathrm{m})$ were stained with hematoxylin and eosin. The degree of airway infiltration by inflammatory cells was scored by two independent investigators by double-blind screening. Peri-bronchiole and peri-vascular inflammation were evaluated using a scoring system of $0-4$, where 0 represents no cells; 1 , a few cells; 2 , a ring of inflammatory cells one cell layer deep; 3 , a ring of inflammatory cells $2-4$ cells deep; and 4 , a ring of inflammatory cells $>4$ cells deep.

\section{Statistical analysis}

GraphPad Prism 5 software was used for statistical analyses. Results are expressed as the mean \pm SEM. The significance of differences between groups was determined using a two tailed unpaired Student's $t$ 
test. A P value $<0.05$ was deemed statistically significant.

\section{Results}

\section{Breg subsets are present in DOCK8-deficient patients and DOCK8 KO mice, but is not capable of producing IL-10}

To investigate the effects of DOCK8 deficiency on homeostasis of Breg cells in humans, we isolated PBMCs from three patients (aged 5-14 years) with confirmed DOCK8 deficiency (Table 1). First, we examined Breg subsets. We found that the percentage and number of $\operatorname{CD} 19^{+} C D 24^{\text {hi }} C D 27^{+} B$ cells within the PBMC population was lower in patients than in age-matched HCs (Fig. 1a), whereas the total B cell

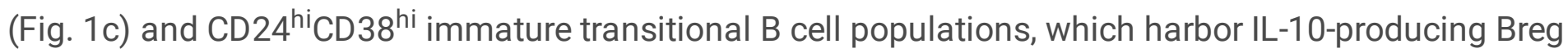
cells, were normal (Fig. 1b). By contrast, the percentage of $C D 1 d^{\text {hi }} C D 5^{+} B$ cells (Fig. $1 \mathrm{~d}$ ) and CD21 ${ }^{\text {hi }} \mathrm{CD} 23^{\text {hi }} \mathrm{CD} 24^{\text {hi }} \mathrm{B}$ cells (T2-MZP) in DOCK8 KO mice was higher than that in controls (Fig. 1e); these cell populations also harbor IL-10-producing Breg cells. Thus, the data suggest that DOCK8 deficiency has little effect on development of $C D 1 d^{\text {hi }} C D 5^{+} B$ cells and T2-MZP cells.

Next, we examined whether DOCK8 expression affects the function of Breg cells in DOCK8-deficient patients and DOCK8 KO mice. PBMCs from patients and HCs were stimulated for $7 \mathrm{~h}$ with LPS plus PMA, ionomycin, and BFA to determine whether the absence of DOCK8 affects IL-10 production by B cells. The results showed that IL- $10^{+} B$ cell numbers in patients were significantly lower than those in HCs; indeed, they were almost absent from patients (Fig. 1f). The percentage and number of IL-10+ $\mathrm{B}$ cells in DOCK8 $\mathrm{KO}$ mice were also lower than those in wild-type mice, especially after immunization with OVA (Fig. 1g). Previously, we reported that, compared with wild-type mice, DOCK8 KO mice show increased serum IgE levels and develop significant airway inflammatory infiltration and airway hyper-responsiveness ${ }^{20}, \mathrm{a}$ finding that is consistent with the data presented above.

\section{DOCK8 deficiency causes an intrinsic defect in Breg cells}

To investigate whether the functional defect in Breg cells from DOCK8 KO mice is intrinsic to these cells, we transferred CD $45.2^{+}$wild-type or DOCK8 KO bone marrow cells plus CD $45.1^{+}$bone marrow cells ( $4 \times 10^{6}$ cells in a 1:1 mixture) into lethally-irradiated (two doses of 550 rads each) wild-type CD45.1 ${ }^{+}$ recipients. Recipient mice were allowed 8 weeks to reconstitute before being challenged with OVA. We found that the percentage and number of CD $45.2^{+} \mathrm{IL}-10^{+} \mathrm{B}$ cells in the spleen of immunized DOCK8 KO chimera mice was significantly lower than that in WT chimera mice (Fig. 2a, b). These results suggest that defective IL-10 production in DOCK8 KO mice is intrinsic to Breg cells.

\section{DOCK8 ${ }^{-/-} \mathrm{CD}^{+} \mathrm{T}$ cells impair IL-10 production by Breg cells}

Breg cells require cognate interactions with IL-21-producing $\mathrm{CD} 4^{+} \mathrm{T}$ cells to secrete IL-10 in vivo [17]. In our own laboratory, we have detected abnormalities in IL-2 $1^{+} \mathrm{CXCR} 5^{+} \mathrm{CD} 4^{+} \mathrm{Tfh}$ cells associated with 
DOCK8 deficiency (data not shown). Therefore, we performed adoptive transfer experiments to confirm the effect of $\mathrm{DOCK8}{ }^{-/-} \mathrm{CD} 4^{+} \mathrm{T}$ cells on IL-10 production by Breg cells. CD4 KO mice received wild-type or DOCK8 KO splenic CD $4^{+}$naïve T cells, followed by challenge with OVA. At 21 days post-immunization with OVA, we analyzed the IL-10 ${ }^{+}$Breg cell population in CD4 KO recipient mice. The percentage of IL- $10^{+}$ Breg cells in CD4 KO mice receiving DOCK $8 \mathrm{KO} C D 4^{+}$naïve $\mathrm{T}$ cells was lower than that in mice receiving wild-type $\mathrm{CD} 4^{+}$naïve $\mathrm{T}$ cells (Fig. 3a). There was no significant difference between two groups with respect to the percentage of $C D 1 d^{\text {hi }}{ }^{C D} 5^{+} B$ cells, T2-MZP cells, or MZB cells (Fig. 3b). Thus, DOCK8 KO $\mathrm{CD}^{+}{ }^{+}$naïve $\mathrm{T}$ cells do not affect the percentages of Breg subsets in recipient mice. These results suggest that loss of DOCK8 from $\mathrm{CD} 4^{+} \mathrm{T}$ cells attenuates IL-10 production by Breg cells in vivo.

\section{Supplementation with IL-21 restores IL-10 production by Breg cells from DOCK8 KO mice both in vitro and in vivo}

To confirm whether reduced IL-21 secretion by $C D 4^{+} T$ cells under conditions of DOCK8 deficiency causes the functional defect in Breg cells, we co-cultured purified splenic B cells $\left(2 \times 10^{6}\right)$ from wild-type or DOCK8 $\mathrm{KO}$ mice with IL-21. After culture for $48 \mathrm{~h}$, the IL-10 concentration in the supernatant was measured in an ELISA. Whereas the percentage of IL- $10^{+}$Breg cells in DOCK8 KO mice was comparable with that in WT mice (Fig 4a), the concentration of IL-10 was higher (Fig. 4b).

Next, to further examine the effect of IL-21 on IL-10 production by Breg cells in vivo, we exposed wild-type or DOCK8 KO mice to intranasal rmIL-21 for 3 or 6 days. Surprisingly, the percentage of IL-10 $0^{+}$Breg cells in the spleen of DOCK8 KO mice was almost comparable with that in wild-type mice (Fig. 4c). Increased production of IL-10 by Breg cells was more obvious in DOCK8 KO mice sensitized with OVA. At the same time, treatment with recombinant IL-21 provided DOCK8 KO mice with marked protection from OVAinduced airway inflammation, accompanied by alleviation of inflammatory infiltration (Fig. 4d, e). Taken together, these suggest that IL-21 plays a critical role in the normal function of Breg cells under conditions of DOCK8 deficiency, and that exogenous L-21 rescues defective IL-10 production by Breg cells in DOCK8 $\mathrm{KO}$ mice.

\section{LPS-driven, not IL-21-driven, STAT3 phosphorylation is defective in Breg cells from DOCK8 KO mice}

Because STAT3 phosphorylation is required for LPS-induced IL-10 production by B cells, we examined this phenomenon in B cells from DOCK8 KO mice stimulated with LPS. We found that levels of STAT3 phosphorylation in DOCK8 KO mice were lower than those in wild-type mice (Fig. 5a, b). Next, we examined STAT3 phosphorylation in CD1 $\mathrm{d}^{\text {hi }} \mathrm{CD}^{+} \mathrm{B}$ cells (B10 cells) from DOCK8 KO mice after stimulation with LPS or IL-21. We found no significant increase in STAT3 phosphorylation in DOCK $8^{-1}$ CD1 $\mathrm{d}^{\text {hi }} \mathrm{CD}^{+} \mathrm{B}$ cells after LPS stimulation (Fig. $5 \mathrm{c}$ ). By contrast, IL-21 caused comparable STAT3 phosphorylation in $\mathrm{CD} 1 \mathrm{~d}^{\text {hi }} \mathrm{CD} 5^{+} \mathrm{B}$ cells (B10 cells) from DOCK8 KO and wild-type mice (Fig. $5 \mathrm{c}$ ).

\section{Discussion}


Here, we present evidence that DOCK8 deficiency impairs IL-10 production by Breg cells due to abnormalities in DOCK8 ${ }^{-/}$IL-21-producing $C D 4^{+} T$ cells. We also show that exogenous IL-21 rescues the function of Breg cells in DOCK8 KO mice both in vitro and in vivo.

B cells play an important role in the pathogenesis of allergic diseases, in particular by secreting lgE. However, several phenotypic subsets have been identified as Breg cells; these cells exert immunosuppressive functions in allergic and inflammatory diseases via release of IL-10 [22, 23]. In murine models of allergic airway disease, Breg deficiency is associated with increased serum IgE levels, increased secretion of type 2 cytokines, and increased eosinophilia [24]. Patients with allergic asthma and rhinitis show a decrease in the percentage of IL-10-secreting CD24hi $C D 27^{+}$Breg cells in response to LPS stimulation $[25,26]$. Injection of IL-10-producing CD9 ${ }^{+}$Breg cells into asthmatic mice normalizes airway inflammation and lung function by inhibiting Th2- and Th17-driven inflammation [27].

In the present study, we developed an OVA-induced allergic asthma model based on DOCK8 KO mice. Model mice showed severe reductions in the percentage of IL-10-producing Breg cells, increased serum IgE levels, and increased inflammatory infiltration compared with wild-type mice and DOCK8 KO mice not exposed to OVA. DOCK8 deficiency is a HIES; therefore, it is associated with a high incidence of allergic disease. Indeed, $71 \%$ of patients have allergic manifestations and $30 \%$ develop asthma [28]. Among the three patients examined in the present study, one patient had food allergies and one had asthma. Due to the small number of enrolled patients, we were unable to test whether the number of IL-10-producing Breg cells differed between patients with and without allergies. Further studies should clarify whether Breg cells are involved in the onset of allergic disease under conditions of DOCK8 deficiency.

Our data also provide insight into the mechanism by which DOCK8 may cause a severe reduction in Breg cell numbers. IL-10 production by Breg cells requires LPS-induced TLR signaling [29]. Nonetheless, IL-21and CD40 dependent cognate interactions with $\mathrm{T}$ cells are also required for IL-10-producing Breg cells to optimally suppress inflammation and autoimmunity [17]. All of these signals are instrumental to Breg expansion and function; thus dysfunction leads to impaired IL-10 production and susceptibility to inflammation and allergy. Because we found impaired expansion of IL- $21^{+} \mathrm{CXCR} 5^{+} \mathrm{CD} 4^{+} \mathrm{Tfh}$ cells in DOCK8 deficiency, we adoptively transferred normal CD4 ${ }^{+} \mathrm{T}$ cells into DOCK8 KO mice and found that this restored the number of IL-10-producing Breg cells, which suggests that abnormalities of DOCK8 $8^{-1-}$ $\mathrm{CD} 4^{+} \mathrm{T}$ cells affect the function of Breg cells. Subsequently, administration of rmIL-21 improved IL-10 production by Breg cells and ameliorated airway inflammation in DOCK8 KO mice. All of these results confirm that IL-21-dependent interactions with T cells play a critical role in the normal function of Breg cells under conditions of DOCK8 deficiency.

IL-21 is a type I cytokine produced mainly by activated CD $4^{+} \mathrm{T}$ cells and natural killer T (NKT) cells [30, 31]. Upon binding to its receptors (IL-21R and a common receptor $y$ chain), IL-21 activates the Janus family tyrosine kinases members JAK1 and JAK3, with subsequent phosphorylation of STAT3 and STAT1 [32]. For functionality, IL-21 mediates maturation of B cells, normal development of T follicular helper cells, and differentiation of Th17 cells [33]. In addition, IL-21 plays a role in the pathogenesis of a series 
of inflammatory diseases. For example, IL-21 has critical immunosuppressive effects in IBD because of its ability to induce IL-10 [34]. As reported previously, TLR-driven STAT3 phosphorylation was defective in B cells from DOCK8-deficient patients, but IL-21 driven STAT3 phosphorylation in B cells from DOCK8deficient patients was comparable with that in healthy controls [5]. Our data suggest that LPS-driven, not IL-21-driven, STAT3 phosphorylation is defective in Breg cells from DOCK8KO mice. Thus, it is possible that IL-21 restores the Breg defect in DOCK8 deficiency.

In summary, we show here that DOCK8 regulates Breg function in both humans and mice. We propose that the absence of Bregs under conditions of DOCK8 deficiency contributes to allergy and chronic inflammation in these patients. Data from this study provide new insight into the potential design of Bregbased or IL-21-based therapeutic strategies for allergic diseases, including asthma in those with DOCK8 deficiency.

\section{Declarations}

\section{Acknowledgments}

We are grateful to the patients and their families for their cooperation with this study. We thank the members of the laboratory for technical assistance.

\section{Ethics approval}

Informed consent was obtained from all individual participants included in the study. This study was conducted in accordance with the tenets of the Declaration of Helsinki and was approved by the Medical Ethics Committee of Children's Hospital of Chongqing Medical University.

\section{Authors' Contributions}

JQ.J. and XD.Z. designed the study and wrote the manuscript; JQ.J., T.Q., L.Z., Q.L., JB.W., RX.D., LN.Z., Q.Z., XY.L., H.W. did the experiments and analyzed the data; T.Q. and XD.Z. followed the patients; all authors reviewed the manuscript before publication.

\section{Funding imformation}

This work was supported by National Natural Science Foundation of China (81803140).

\section{Consent to participate}

Written informed consent was obtained from individual or guardian participants.

\section{Consent to publication}

Written informed consent was obtained from individual or guardian participants.

\section{Availability of data and material}


The data that support the findings of this study are available form the corresponding authors, XD.Z upon reasonable request.

\section{Code availability}

Not applicable.

\section{Disclosure of Conflicts of Interest}

The authors declare no conflicts of interest related to the content of the manuscript.

\section{References}

1. Tangye SG, Pillay B, Randall KL, Avery DT, Phan TG, Gray P et al. Dedicator of cytokinesis 8-deficient CD4+ T cells are biased to a TH2 effector fate at the expense of TH1 and TH17 cells. The Journal of allergy and clinical immunology. 2016. doi:10.1016/j.jaci.2016.07.016.

2. Zhang Q, Jing H, Su HC. Recent Advances in DOCK8 Immunodeficiency Syndrome. Journal of clinical immunology. 2016;36(5):441-9. doi:10.1007/s10875-016-0296-z.

3. Ponsford MJ, Klocperk A, Pulvirenti F, Dalm V, Milota T, Cinetto F et al. Hyper-IgE in the allergy clinic-when is it primary immunodeficiency? Allergy. 2018;73(11):2122-36. doi:10.1111/all.13578.

4. Keles S, Charbonnier LM, Kabaleeswaran V, Reisli I, Genel F, Gulez N et al. Dedicator of cytokinesis 8 regulates signal transducer and activator of transcription 3 activation and promotes $\mathrm{TH} 17$ cell differentiation. The Journal of allergy and clinical immunology. 2016;138(5):1384-94 e2. doi:10.1016/j.jaci.2016.04.023.

5. Jabara HH, McDonald DR, Janssen E, Massaad MJ, Ramesh N, Borzutzky A et al. DOCK8 functions as an adaptor that links TLR-MyD88 signaling to $B$ cell activation. Nature immunology. 2012;13(6):612-20. doi:10.1038/ni.2305.

6. Randall KL, Lambe T, Johnson AL, Treanor B, Kucharska E, Domaschenz H et al. Dock8 mutations cripple B cell immunological synapses, germinal centers and long-lived antibody production. Nature immunology. 2009;10(12):1283-91. doi:10.1038/ni.1820.

7. aan de Kerk DJ, van Leeuwen EMM, Jansen MH, van den Berg JM, Alders M, Vermont CL et al. Aberrant humoral immune reactivity in DOCK8 deficiency with follicular hyperplasia and nodal plasmacytosis. Clinical immunology. 2013;149(1):25-31.

8. Shen P, Roch T, Lampropoulou V, O'Connor RA, Stervbo U, Hilgenberg E et al. IL-35-producing B cells are critical regulators of immunity during autoimmune and infectious diseases. Nature. 2014;507(7492):366-70. doi:10.1038/nature12979.

9. Parekh VV, Prasad DVR, Banerjee PP, Joshi BN, Kumar A, Mishra GC. B Cells Activated by Lipopolysaccharide, But Not By Anti-Ig and Anti-CD40 Antibody, Induce Anergy in CD8+ T Cells: Role of TGF-?1. Journal of immunology. 2003;170(12):5897-911. 
10. Carter NA, Vasconcellos R, Rosser EC, Tulone C, Muoz-Suano A, Kamanaka M et al. Mice lacking endogenous IL-10-producing regulatory $B$ cells develop exacerbated disease and present with an increased frequency of Th1/Th17 but a decrease in regulatory T cells. Journal of immunology. 2011;186(10):5569.

11. Yanaba K, Bouaziz JD, Haas KM, Poe JC, Fujimoto M, Tedder TF. A regulatory B cell subset with a unique $\mathrm{CD} 1 \mathrm{dhiCD} 5+$ phenotype controls $\mathrm{T}$ cell-dependent inflammatory responses. Immunity. 2008;28(5):639-50. doi:10.1016/j.immuni.2008.03.017.

12. Bankoti R, Gupta K, Levchenko A, Stager S. Marginal Zone B Cells Regulate Antigen-Specific T Cell Responses during Infection. Journal of immunology. 2012;188(8):3961-71.

13. Interleukin-10-Producing Plasmablasts Exert Regulatory Function in Autoimmune Inflammation. 2014.

14. Blair PA, Norena LY, Flores-Borja F, Rawlings DJ, Isenberg DA, Ehrenstein MR et al. CD19(+)CD24(hi)CD38(hi) B cells exhibit regulatory capacity in healthy individuals but are functionally impaired in systemic Lupus Erythematosus patients. Immunity. 2010;32(1):129-40. doi:10.1016/j.immuni.2009.11.009.

15. Yanaba K, Bouaziz JD, Matsushita T, Tsubata T, Tedder TF. The development and function of regulatory B cells expressing IL-10 (B10 cells) requires antigen receptor diversity and TLR signals. Journal of immunology. 2009;182(12):7459-72. doi:10.4049/jimmunol.0900270.

16. Candando KM, Lykken JM, Tedder TF. B10 cell regulation of health and disease. Immunological Reviews. 2014;259(1):259-72.

17. Yoshizaki A, Miyagaki T, DiLillo DJ, Matsushita T, Horikawa M, Kountikov El et al. Regulatory B cells control T-cell autoimmunity through IL-21-dependent cognate interactions. Nature. 2012;491(7423):264-8. doi:10.1038/nature11501.

18. Qin T, An Y, Liu C, Wu J, Dai R, Liu D et al. Novel DOCK8 gene mutations lead to absence of protein expression in patients with hyper-IgE syndrome. Immunologic research. 2016;64(1):260-71. doi:10.1007/s12026-015-8745-y.

19. Sun X, Wang J, Qin T, Zhang Y, Liu C. Dock8 regulates BCR signaling and activation of memory B cells via WASP and CD19. Blood Advances. 2018;2(4):401.

20. Wu J, Zhang S, Qin T, Jiang J, Liu Q, Zhang L et al. IL-21 alleviates allergic asthma in DOCK8knockout mice. Biochemical \& Biophysical Research Communications. 2018:S0006291X18309720.

21. Hiromura Y, Kishida T, Nakano H, Hama T, Imanishi J, Hisa Y et al. IL-21 Administration into the Nostril Alleviates Murine Allergic Rhinitis. Journal of immunology. 2007;179(10):7157-65.

22. Singh A, Carson WF, Secor ER, Guernsey LA, Flavell RA, Clark RB et al. Regulatory Role of B Cells in a Murine Model of Allergic Airway Disease. Journal of immunology. 2008;180(11):7318-26.

23. Willem VDV, Stanic B, Wirz OF, Jansen K, Globinska A, Akdis M. Role of regulatory B cells in immune tolerance to allergens and beyond. Journal of Allergy \& Clinical Immunology. 2016;138(3):654-65.

24. Amu S, Saunders SP, Kronenberg M, Mangan NE, Atzberger A, Fallon PG. Regulatory B cells prevent and reverse allergic airway inflammation via FoxP3-positive T regulatory cells in a murine model. The 
Journal of allergy and clinical immunology. 2010;125(5):1114-2147483647.

25. Lucin EPMvdV, Mlejnek E, Ozir-Fazalalikhan A, Bonas MJ, Hermelijn HS. CD24(hi) CD27(+) B cells from allergic asthma patients have impaired regulatory activity in response to LPS. Clinical \& Experimental Allergy Journal of the British Society for Allergy \& Clinical Immunology. 2013;44(4).

26. Sarah, Salomon, Caroline, Guignant, Pierre, Morel et al. Th17 and CD24hiCD27+ regulatory B lymphocytes are biomarkers of response to biologics in rheumatoid arthritis. Arthritis Research \& Therapy. 2017.

27. F, Braz, J, Chesne, M, Durand et al. A regulatory CD9+B-cell subset inhibits HDM-induced allergic airway inflammation. Allergy. 2015.

28. Aydin SE, Kilic SS, Aytekin C, Kumar A, Porras O, Kainulainen L et al. DOCK8 deficiency: clinical and immunological phenotype and treatment options - a review of 136 patients. Journal of clinical immunology. 2015;35(2):189-98. doi:10.1007/s10875-014-0126-0.

29. Lampropoulou V, Hoehlig K, Roch T, Neves P, Gomez EC, Sweenie CH et al. TLR-Activated B Cells Suppress T Cell-Mediated Autoimmunity. Journal of immunology. 2008;180(7):4763-73.

30. Jonathan M, Coquet, Martijn J, Schuijs, Mark J, Smyth et al. Interleukin-21-Producing CD4+ T Cells Promote Type 2 Immunity to House Dust Mites. Immunity. 2015.

31. Parrish-Novak J. Interleukin 21 and its receptor are involved in NK cell expansion and regulation of lymphocyte function. Nature. 2000;408(6808):57-63.

32. Novy P, Huang X, Leonard WJ, Yang Y. Intrinsic IL-21 Signaling Is Critical for CD8 T Cell Survival and Memory Formation in Response to Vaccinia Viral Infection. Journal of immunology. 2011;186(5):2729-38.

33. Habib T, Nelson A, Kaushansky K. IL-21: a novel IL-2-family lymphokine that modulates B, T, and natural killer cell responses. The Journal of allergy and clinical immunology. 2003;112(6):1033-45.

34. Fusco DD, Izzo R, Figliuzzi MM, Pallone F, Monteleone G. IL-21 as a therapeutic target in inflammatory disorders. Expert Opinion on Therapeutic Targets. 2014;18(11):1329-38.

\section{Table}

Table 1. Clinical features of DOCK8-deficient patients 


\begin{tabular}{|c|c|c|c|}
\hline Features & P1/Male & P2/Female & P3/Male \\
\hline Age at onset & $6 y$ & 1 y $7 \mathrm{~m}$ & $3 y$ \\
\hline Age at diagnosis & $14 y$ & 5 y $8 \mathrm{~m}$ & $9 y$ \\
\hline DOCK8 mutation & $\begin{array}{l}\text { Exon } 11 \text { homozygous } \\
\text { deletion }+ \text { Exon } 12-33 \\
\text { heterozygous deletion }\end{array}$ & $\begin{array}{l}\text { Exon2 homozygous } \\
\text { deletion + Exon1, } 3-39 \\
\text { heterozygous deletion }\end{array}$ & $\begin{array}{l}\text { c.1278- } \\
\text { 1279delTG, } \\
\text { p.V427fsX435 }\end{array}$ \\
\hline $\begin{array}{l}\text { DOCK8 protein } \\
\text { expression }\end{array}$ & - & - & - \\
\hline Atopy & $\begin{array}{l}\text { Eczema, } \\
\text { asthma }\end{array}$ & Eczema, & $\begin{array}{l}\text { Eczema, } \\
\text { food allergy }\end{array}$ \\
\hline Immunodeficiency & $\begin{array}{l}\text { Respiratory infection, otitis } \\
\text { media, sinusitis, stomatitis }\end{array}$ & $\begin{array}{l}\text { Respiratory infection, otitis } \\
\text { media, stomatitis }\end{array}$ & $\begin{array}{l}\text { Respiratory } \\
\text { infection, otitis } \\
\text { media, stomatitis }\end{array}$ \\
\hline $\begin{array}{l}\text { Autoimmune } \\
\text { disease }\end{array}$ & - & - & $\begin{array}{l}\text { Autoimmune } \\
\text { hemolytic anemia }\end{array}$ \\
\hline Malignancy & - & - & - \\
\hline $\begin{array}{l}\text { Etiology of } \\
\text { infections }\end{array}$ & HSV & EBV & EBV \\
\hline $\begin{array}{l}\text { Molluscum } \\
\text { contagiosum }\end{array}$ & + & + & + \\
\hline
\end{tabular}

HSV, herpes simplex virus; EBV, Epstein-Barr virus.

\section{Figures}


a

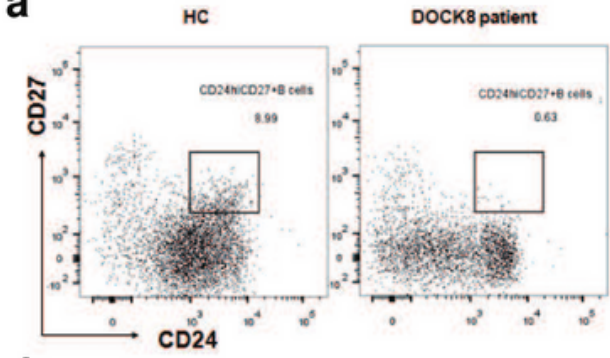

d
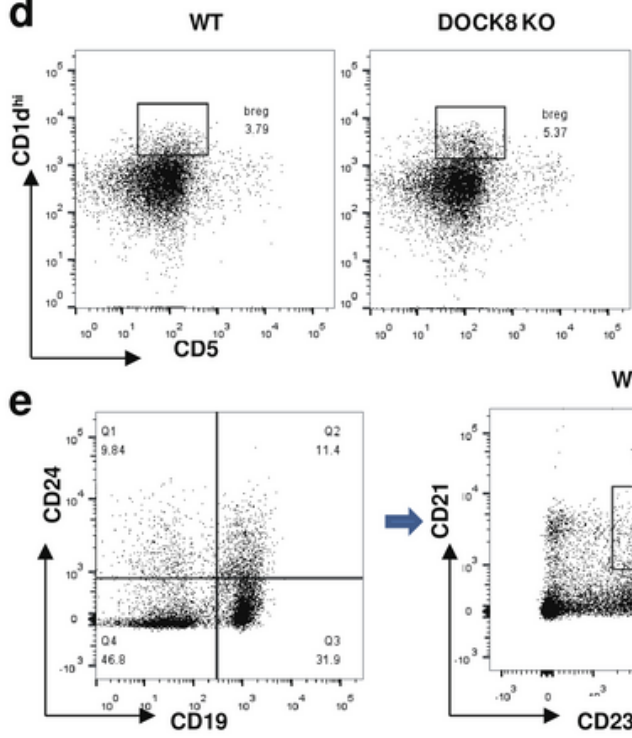

f

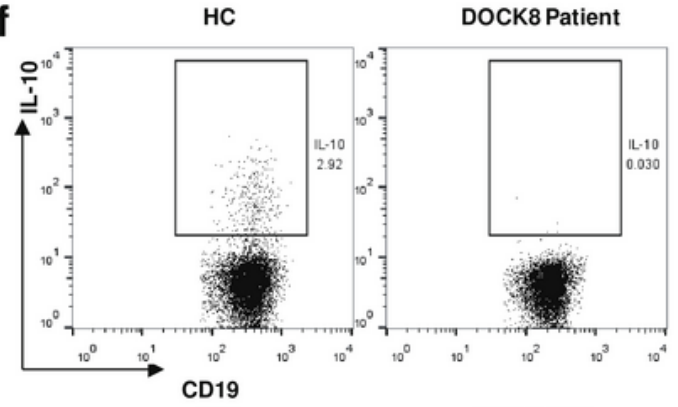

$\mathbf{g}$

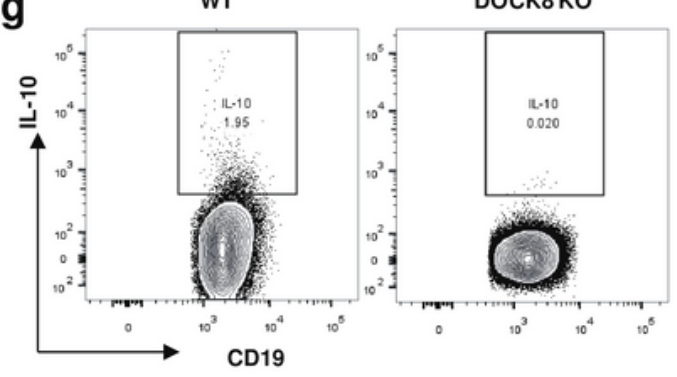

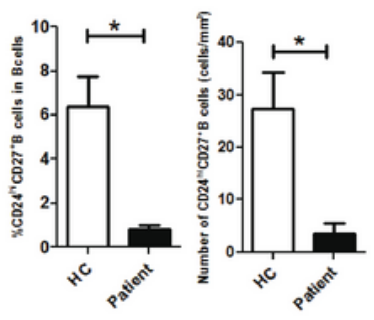

b

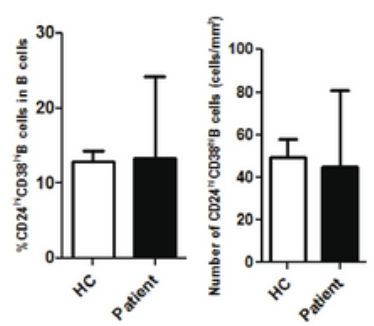

C

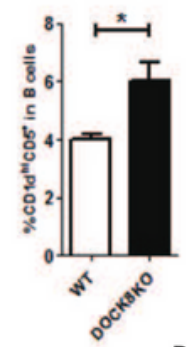

DOCK8 KO
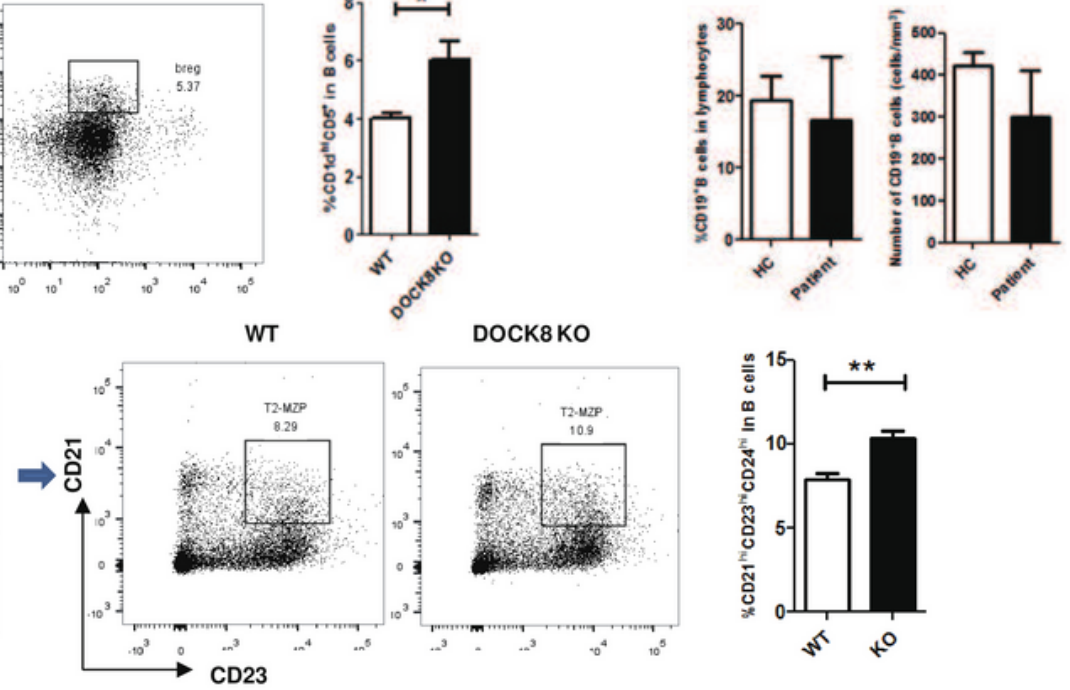
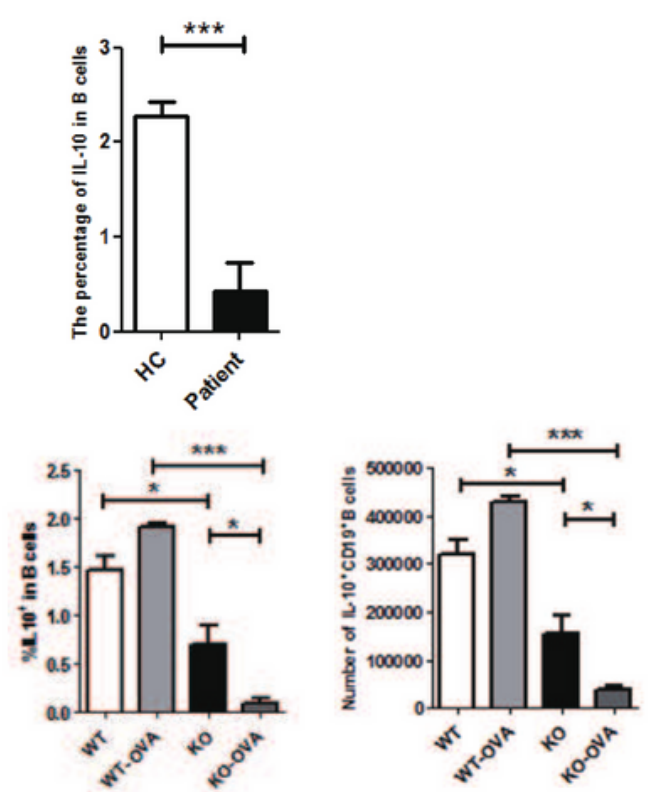

\section{Figure 1}

Reduced percentage of IL-10-producing Breg cells in DOCK8-deficient patients and DOCK8 KO mice. (a) Percentage and number of CD19+CD24hiCD27+ B cells, (b) CD19+CD24hiCD38hi B cells, and (c) CD19+ $B$ cells in HCs and DOCK8-deficient patients ( $n=3$ for both). (d) CD19+CD5+CD1dhi B cell and (e) CD19+CD21hiCD23hiCD24hiT2-MZP cell populations in the spleen of wild-type and DOCK8 KO mice. (f) The percentage of IL-10+CD19+ Breg cells in HCs and DOCK8-deficient patients (n=3 for both). (g) 
Percentage of IL-10+CD19+ Breg cells in the spleen of wild-type and DOCK8 KO mice immunized (or not) with OVA. ${ }^{*} P<0.05,{ }^{*} \mathrm{P}<0.01$, and ${ }^{* * * P}<0.001$.
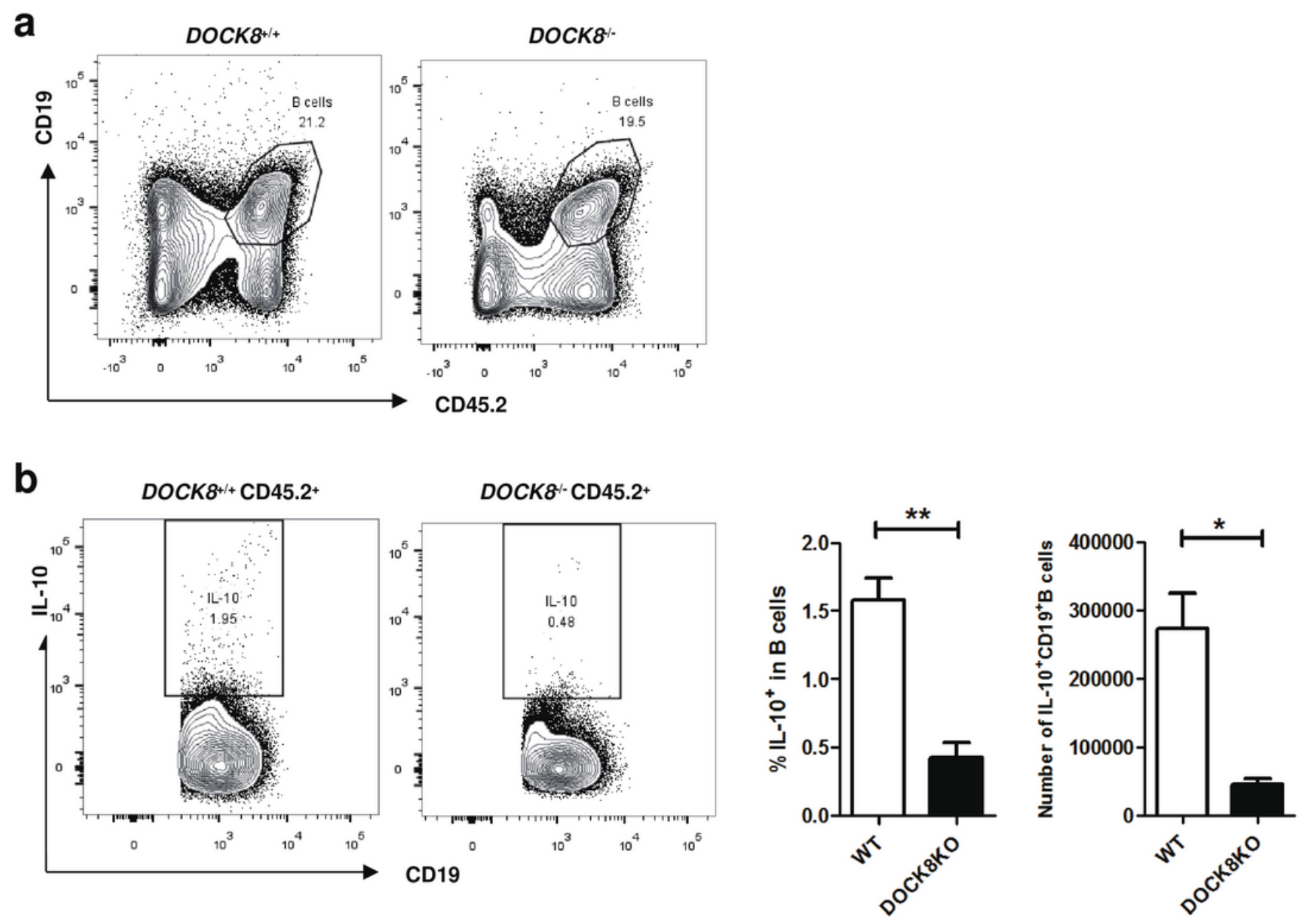

Figure 2

Defective IL-10 production in DOCK8 KO mice is intrinsic to Breg cells. ( $a$ and b) CD45.2+ wild-type or DOCK8 KO bone marrow cells were transferred intravenously into lethally-irradiated CD45.1+ wild-type recipients prior to immunization with OVA. Flow cytometry analysis of CD45.2+CD19+IL-10+B cells in the splenocyte population from $C D 45.1+$ chimeric mice. ${ }^{*} \mathrm{P}<0.05,{ }^{*} \mathrm{P}<0.01$. 

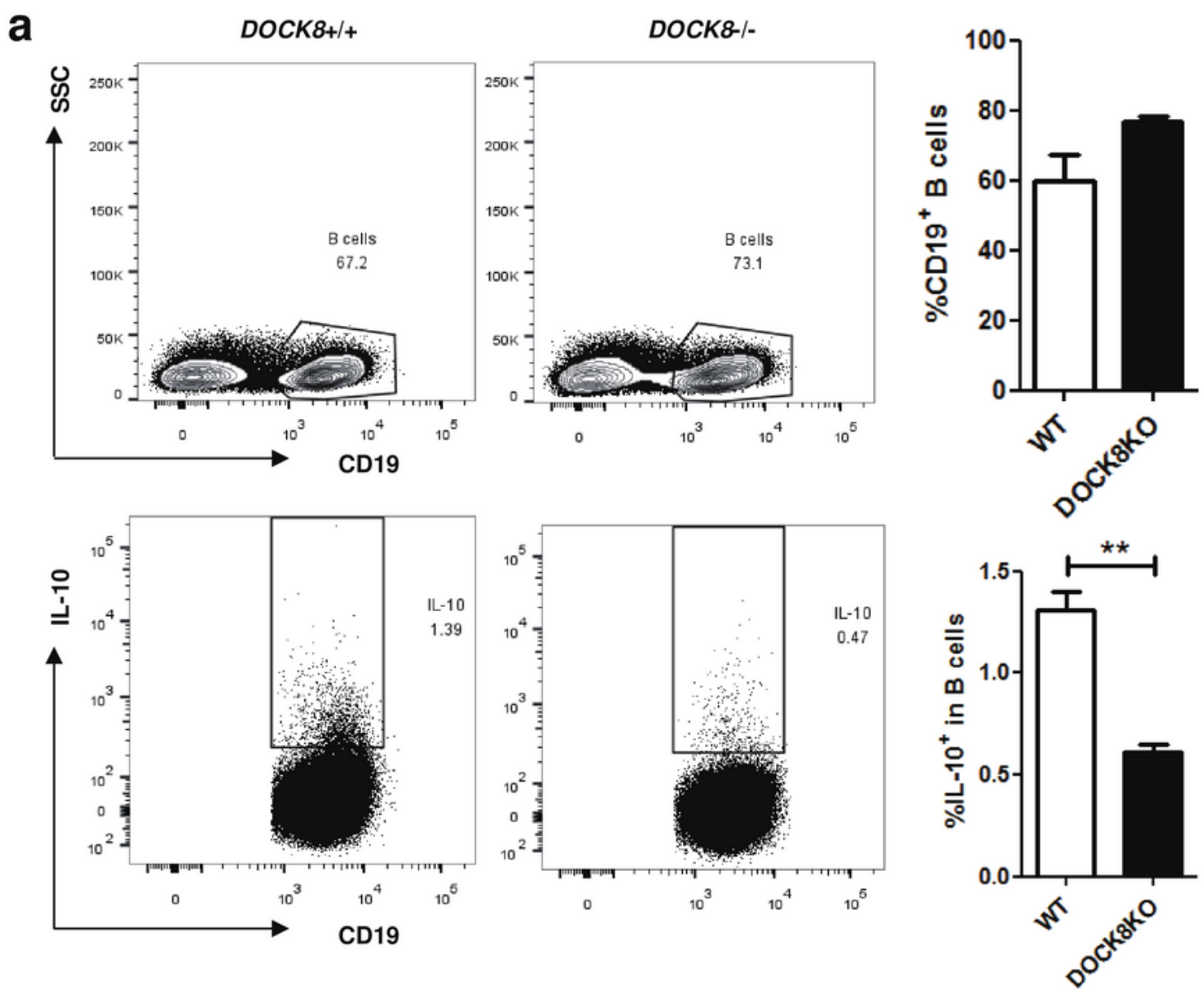

b

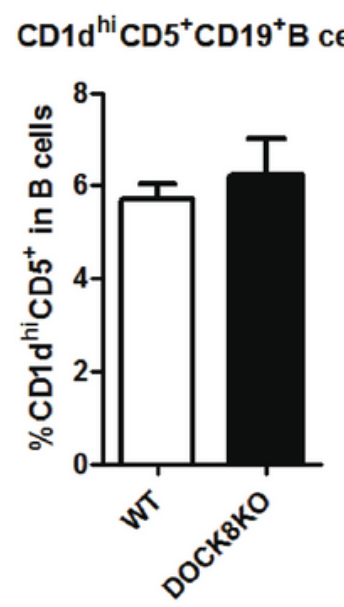

T2-MZP

MZB
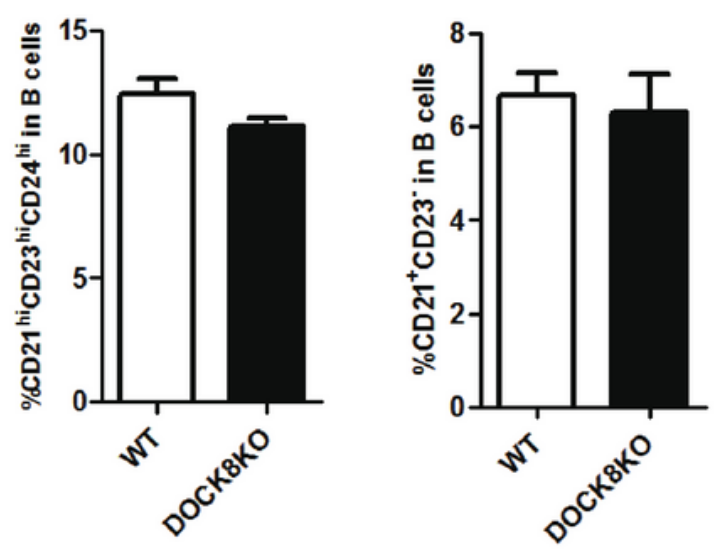

Figure 3

DOCK8-/- CD4+ T cell impairs IL-10 production by Breg cells. (a) Splenic CD4+ naïve T cells from wildtype or DOCK8 KO mice were transferred into CD4KO mice prior to immunization with OVA. The percentage of IL-10+ Breg cells in CD4 KO mice receiving DOCK8 KO CD4+ naïve T cells was lower than that in mice receiving wild-type CD4+ naïve T cells. (b) DOCK8-/- CD4+ T cells have no effect on the percentages of different Breg subtypes. ${ }^{*} \mathrm{P}<0.05$. 

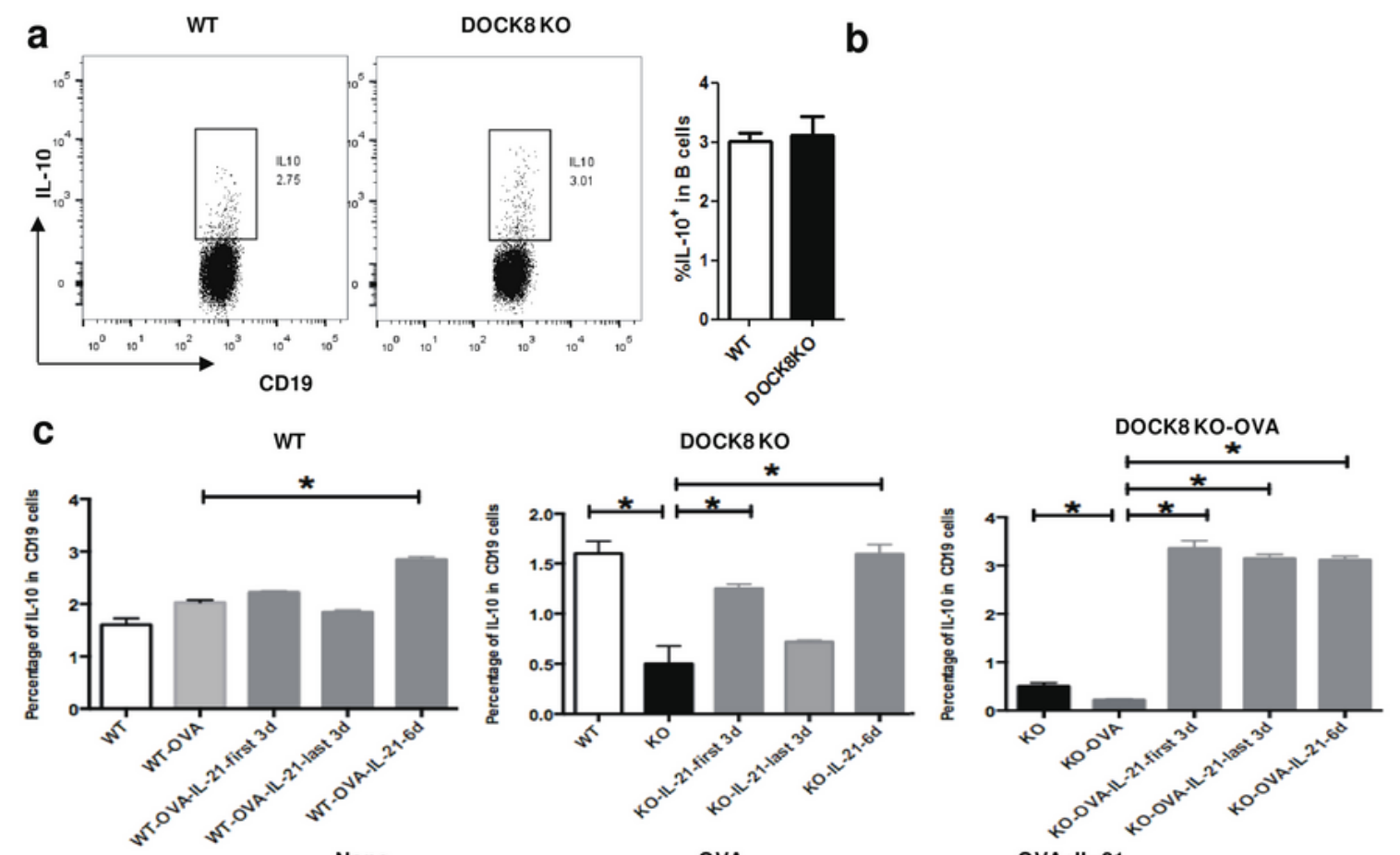

d

None

OVA+IL-21
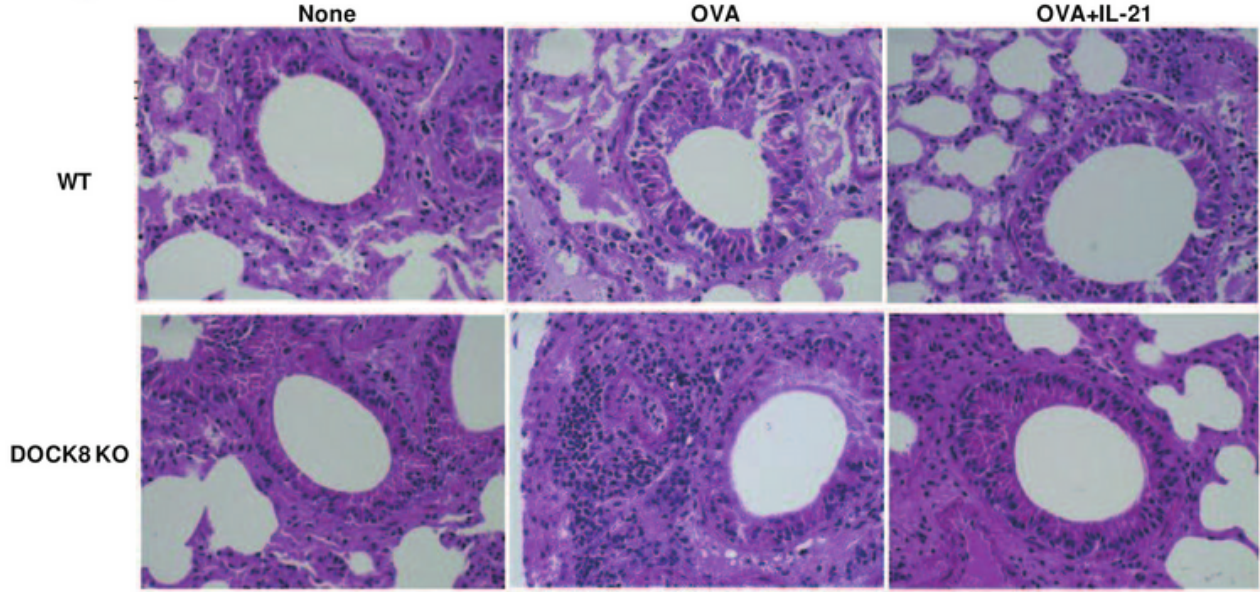

e

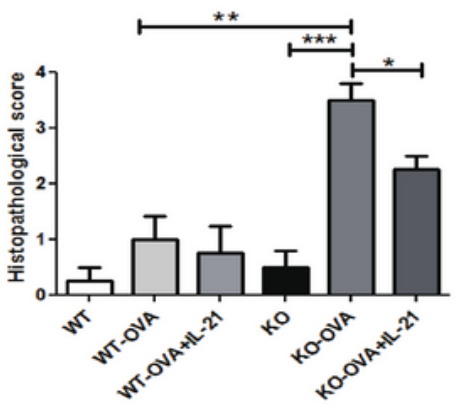

\section{Figure 4}

Exogenous IL-21 rescues IL-10 production by Breg cells in DOCK8 KO mice both in vitro and in vivo. (a) Splenic B cells isolated from wild-type or DOCK8 KO mice were co-cultured with IL-21 for 48 hours and the percentage of CD19+IL-10+ B cells was analyzed by FCM. (b) The IL-10 concentration in the supernatant was measured in an ELISA. (c) The percentage of CD19+IL-10+ Breg cells in wild-type and DOCK8 KO mice administered $20 \mathrm{ng}$ of rmlL-21 into the nostrils daily for 3 or 6 days and then immunized with OVA. 
(d) Representative images of $\mathrm{H} \& \mathrm{E}$ stained lung tissue from each genotype in the OVA-induced allergic asthma model. (e) Histopathological score for airway inflammation. ${ }^{\star} \mathrm{P}<0.05,{ }^{\star} \mathrm{P}<0.01$, and ${ }^{\star \star \star} \mathrm{P}<$ 0.001 .

a
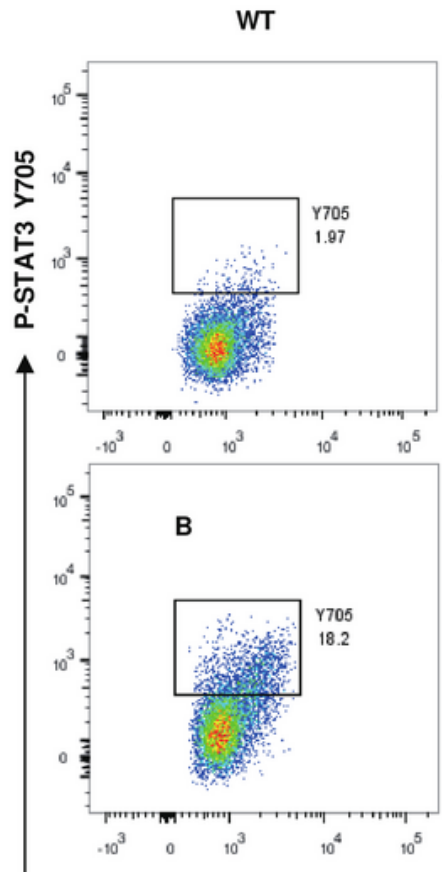

DOCK8KO

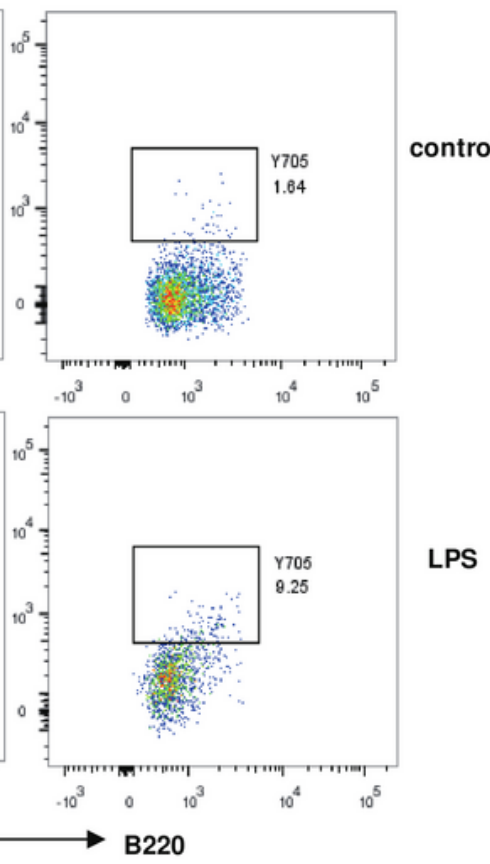

b

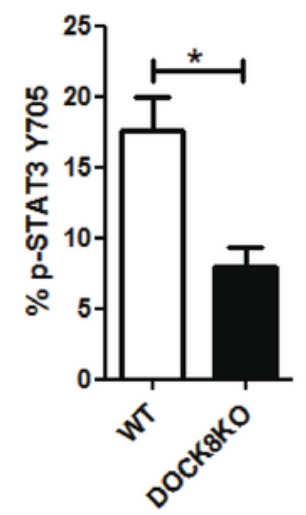

C

IL-21

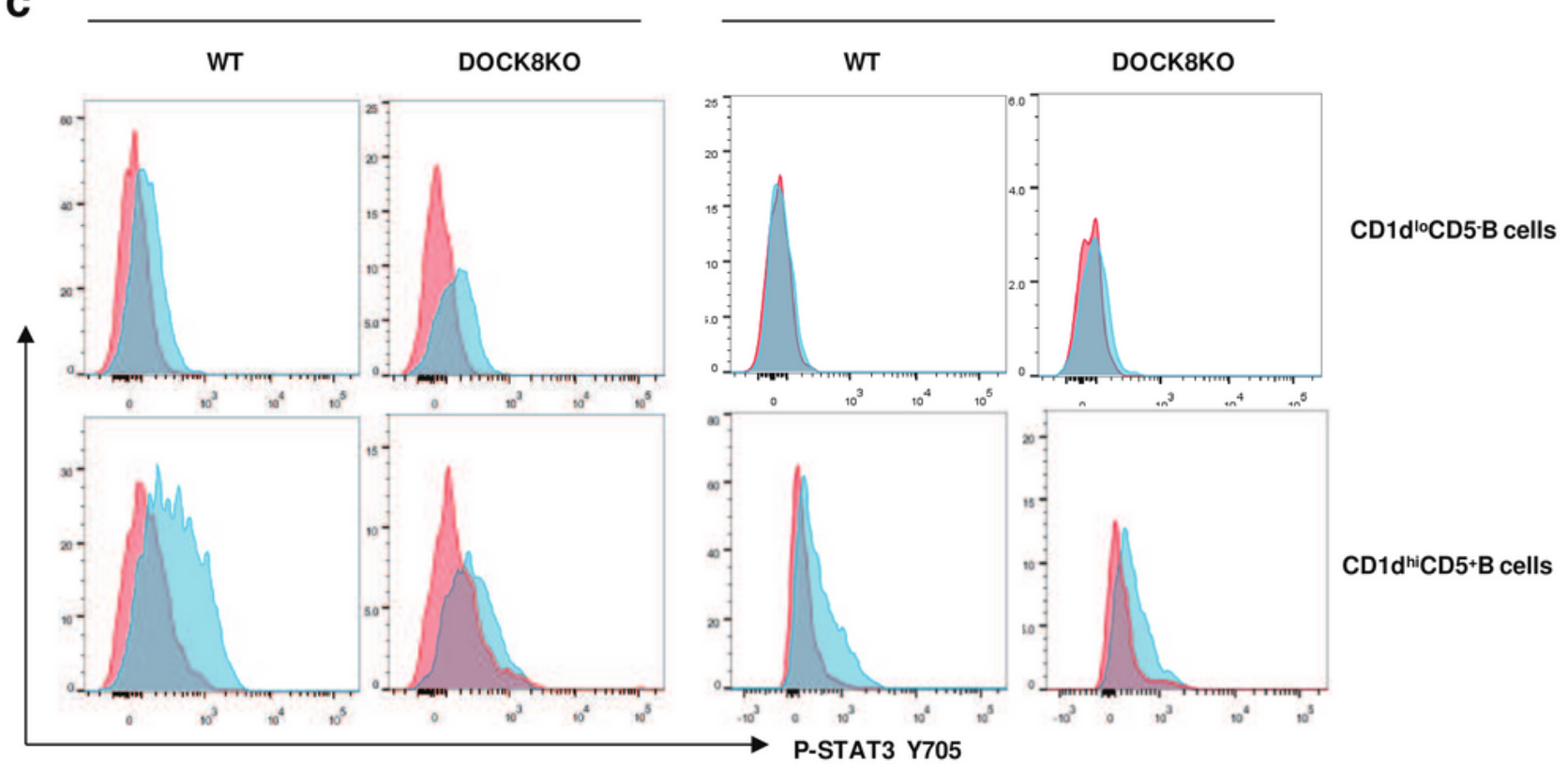

Figure 5

LPS-induced STAT3 phosphorylation is defective in Breg cells from DOCK8 KO mice but not IL-21-induced STAT3 phosphorylation. (a and b) STAT3 phosphorylation on Y705 in splenic B cells from DOCK8 KO 
mice and wild-type mice after 3 hours of LPS stimulation. (c) STAT3 phosphorylation in CD5+CD1dhi B cells and CD5-CD1dlo B cells after stimulation with LPS for 3 hours or rmIL-21 for 30 minutes. ${ }^{*} P<0.05$. 